\title{
Myositis, rhabdomyolysis and severe hypercalcaemia in a body builder
}

\author{
Ravikumar Ravindran'1, Justyna Witczak', Suhani Bahl'1, \\ Lakdasa D K E Premawardhana $\mathbb{D}^{1,2}$ and Mohamed Adlan'
}

1Section of Endocrinology, YYF Hospital, Ystrad Fawr Way, Caerphilly, UK and ${ }^{2}$ Centre for Endocrine and Diabetes Sciences, University Hospital of Wales, Cardiff, UK
Correspondence should be addressed to R Ravindran Email

dr.ravimrcp@gmail.com

\section{Summary}

A 53-year-old man who used growth hormone (GH), anabolic steroids and testosterone (T) for over 20 years presented with severe constipation and hypercalcaemia. He had benign prostatic hyperplasia and renal stones but no significant family history. Investigations showed - (1) corrected calcium (reference range) $3.66 \mathrm{mmol} / \mathrm{L}$ (2.2-2.6), phosphate $1.39 \mathrm{mmol} / \mathrm{L}$ (0.80-1.50), and PTH $2 \mathrm{pmol} / \mathrm{L}$ (1.6-7.2); (2) urea $21.9 \mathrm{mmol} / \mathrm{L}$ (2.5-7.8), creatinine $319 \mathrm{mmol} / \mathrm{L}$ (58-110), eGFR $18 \mathrm{~mL} / \mathrm{min}(>90)$, and urine analysis (protein 4+, glucose 4+, red cells 2+); (3) creatine kinase $7952 \mathrm{U} / \mathrm{L}$ (40-320), positive anti Jo-1, and Ro-52 antibodies; (4) vitamin D 46 mmol/L (30-50), vitamin D3 29 pmol/L (55-139), vitamin A $4.65 \mathrm{mmol} / \mathrm{L}$ (1.10-2.60), and normal protein electrophoresis; (5) normal CT thorax, abdomen and pelvis and MRI of muscles showed 'inflammation', myositis and calcification; (6) biopsy of thigh muscles showed active myositis, chronic myopathic changes and mineral deposition and of the kidneys showed positive CD3 and CD45, focal segmental glomerulosclerosis and hypercalcaemic tubular changes; and (7) echocardiography showed left ventricular hypertrophy (likely medications and myositis contributing), aortic stenosis and an ejection fraction of $44 \%$, and MRI confirmed these with possible right coronary artery disease. Hypercalcaemia was possibly multifactorial - (1) calcium release following myositis, rhabdomyolysis and acute kidney injury; (2) possible primary hyperparathyroidism (a low but detectable PTH); and (3) hypervitaminosis A. He was hydrated and given pamidronate, mycophenolate and prednisolone. Following initial biochemical and clinical improvement, he had multiple subsequent admissions for hypercalcaemia and renal deterioration. He continued taking GH and T despite counselling but died suddenly of a myocardial infarction.

\section{Learning points:}

- The differential diagnosis of hypercalcaemia is sometimes a challenge.

- Diagnosis may require multidisciplinary expertise and multiple and invasive investigations.

- There may be several disparate causes for hypercalcaemia, although one usually predominates.

- Maintaining 'body image' even with the use of harmful drugs may be an overpowering emotion despite counselling about their dangers.

\section{Background}

Primary hyperparathyroidism, drugs and malignant disease are common causes of hypercalcaemia in secondary care. However, granulomatous disorders, immobility, familial hypocalciuric hypercalcaemia (FHH) and vitamin D excess are also responsible occasionally
$(1,2,3)$. Inflammatory myositis and rhabdomyolysis may occasionally cause hypercalcaemia severe enough to require treatment. In this situation, calcium phosphate complexes laid down during the oliguric phase of acute kidney injury (AKI) are thought to be released during the 
polyuric phase of AKI causing significant hypercalcaemia (4). Subjects who present with these and other rarer causes of hypercalcaemia, either alone or in combination, are often a diagnostic and management dilemma. Multiple, complicated and sometimes invasive investigations may need to be done in order to diagnose a cause for their hypercalcaemia and to direct therapy. Their management often requires multidisciplinary expertise.

\section{Case presentation}

A 53-year-old retired fire-fighter, who was an obsessive body builder, presented with severe constipation of many weeks' duration. He had no osmotic symptoms, bone or muscle pains, and denied other symptoms. He volunteered no erectile dysfunction or loss of libido and passed normal quantities of urine without discoloration or symptoms of renal dysfunction. His appetite and weight were normal, and he took lansoprazole and tamsulosin only without over-the-counter medications. However, he admitted to the use of growth hormone (GH) 2 IU/day and testosterone (T) $350 \mathrm{mg} /$ day on a '6-weeks on and 4 -weeks off' regime for over 20 years. He did not smoke or consume alcohol. There was no significant family history and he had no children of his own.

On examination, he appeared well, had mild ankle oedema, but no clubbing, jaundice or lymphadenopathy. He had hard globular masses under both axillae which were difficult to characterize. His limb girdle muscles were bulky, well developed and non-tender. Muscle power in all muscle groups was normal. Systems examination was unremarkable and, in particular, there were no skin abnormalities, hepatosplenomegaly or palpable abdominal masses.

\section{Investigation}

Investigations at presentation and subsequently showed the following -

(i) Biochemistry - (1) Corrected calcium $3.66 \mathrm{mmol} / \mathrm{L}$ (2.2-2.6), phosphate $1.39 \mathrm{mmol} / \mathrm{L}(0.8-1.5)$, PTH $2 \mathrm{mmol} / \mathrm{L}$ (1.6-7.2); (2) serum urea $21.9 \mathrm{mmol} / \mathrm{L}$ (2.5-7.8), serum creatinine $319 \mathrm{mmol} / \mathrm{L}(58-110)$, eGFR $18 \mathrm{~mL} / \mathrm{min}$ (>90) (79 for about 5 years and 43 for about 4 years before admission); (3) urine protein $4+$, protein:creatinine ratio $493 \mathrm{mg} / \mathrm{mmol}($ normal $<50$ ) (previous $24 \mathrm{~h}$-protein output - $6.54 \mathrm{~g} / 24 \mathrm{~h}(<0.2)$ ); serum albumin 29-33 g/L; (4) serum creatine kinase (CK) $7952 \mathrm{U} / \mathrm{L}$ (40-320); (5) free thyroxine $8.9 \mathrm{pmol} / \mathrm{L}$
(9-19.1), TSH 1.76 mU/L (0.3-4.4); (6) no abnormal bands on protein electrophoresis; (7) vitamin $\mathrm{D}$ $46 \mathrm{nmol} / \mathrm{L}(>30)$; 1,25 vitamin D3 29 pmol/L (55139); Vitamin A $4.65 \mathrm{~mol} / \mathrm{L}$ (1.1-2.6); (8) Angiotensin converting enzyme (ACE) $28 \mathrm{U} / \mathrm{L}(8-52)$.

(ii) Imaging - (1) ultrasound scans - kidneys, ureters and bladder showed no intrinsic abnormalities or obstructive uropathy and the thyroid and parathyroid glands were normal; (2) CT and MRI scans - thorax, abdomen and pelvis were normal without lymphadenopathy and whole body MRI scans showed muscle oedema and appearances of 'myositis' with areas of calcification and ossification within the muscle bellies of the upper and lower limbs (Figs 1 and 2); (3) echocardiography showed concentric left ventricular hypertrophy (LVH), moderate bicuspid aortic stenosis (AS) with a peak gradient of $39 \mathrm{mmHg}$ and an ejection fraction (EF) of 44-53\%; (6) cardiac MRI confirmed AS with severely impaired LV function. Slightly worse function inferiorly suggested right coronary artery disease. Appearances suggested that his medications and myositis contributed to LVH.

(iii) Autoimmune antibodies - (1) Anti Jo-1 and anti Ro antibodies were positive; (2) However, other myositis specific and myositis associated antibodies (anti Mi 2a and 2b, SRP, EJ, OJ, PL7, PL 12, PMScl75 and PMScl100, Ro 52, SRP, Ku, SAE 1, NPX 2, MDA 5 and TIF-1g), and anti-dsDNA, ENA and ANCA were

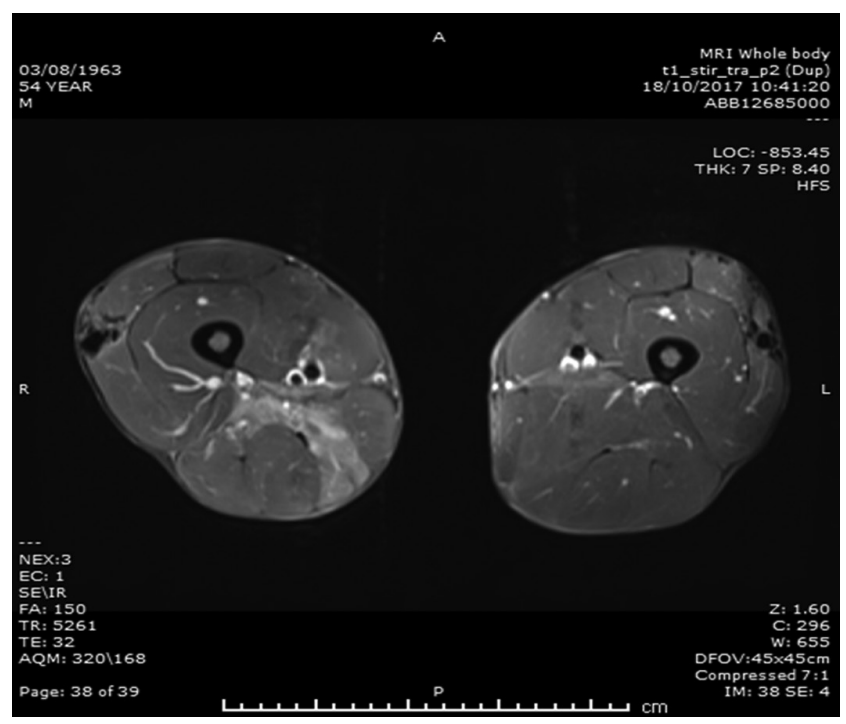

Figure 1

MRI scans of thighs. Transverse views of MRI scans of both thighs. Solid white arrows indicate areas of calcification; solid black arrow indicates areas of myositis and oedema. 


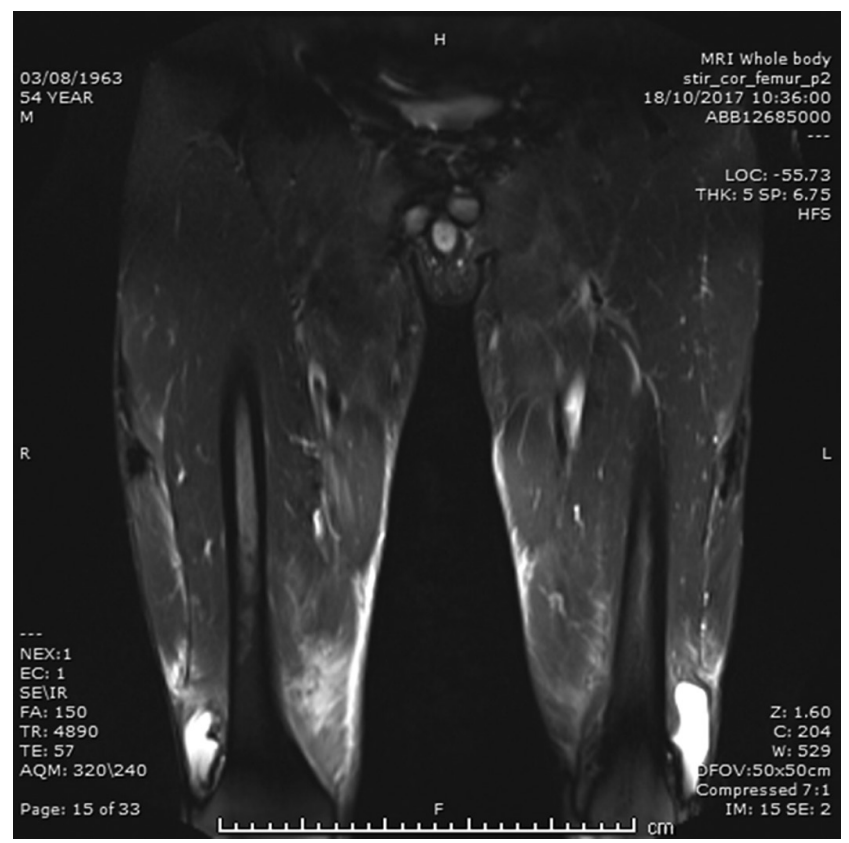

Figure 2

MRI scans of thighs. Longitudinal views of MRI scans of both thighs. Solid white arrows indicate areas of calcification; solid black arrow indicates areas of myositis and oedema.

negative; (3) complement $\mathrm{C} 3$ and $\mathrm{C} 4$ were within the reference range; and (4) anti-liver, anti-GBM and anti-cardiolipin (IgM and $\operatorname{IgG}$ ) antibodies were also negative.

(iv) Histopathology - (1) thigh muscle biopsy (vastus medialis and lateralis) showed an active inflammatory myositis with chronic myopathic changes and mineral deposition. Sections were positive for markers of inflammation (CD3) and fiber formation (CD45) confirming an acute on chronic inflammatory process. Special stains and paraffin sections ruled out amyloid and vasculitis within the muscle. There were no visible granulomata. (2) kidney - most glomeruli were globally sclerosed and some glomeruli showed segmental sclerosis, there was moderate to severe tubular atrophy with interstitial fibrosis and a moderate chronic inflammatory infiltrate. The surviving proximal tubules showed mild dilatation consistent with hyperplasia and mild acute tubular injury with occasional foci of calcification. A diagnosis of focal segmental glomerulosclerosis (FSGS) was made with no specific features to determine the cause -tubular interstitial changes were thought to be due to hypercalcemia.

\section{Treatment}

At his first admission the following diagnoses were made (1) likely non-PTH mediated severe hypercalcaemia likely due to acute myositis and rhabdomyolysis; (2) acute kidney injury (AKI) with significant proteinuria complicating chronic kidney disease. Subsequent investigations pointed to contributions to hypercalcaemia from (1) likely primary hyperparathyroidism and (2) hypervitaminosis A. Acute myositis and rhabdomyolysis were of unknown aetiology but likely iatrogenic, non-traumatic or idiopathic.

He was rapidly rehydrated with normal saline according to protocols for hypercalcaemia and AKI and i.v. pamidronate $60 \mathrm{mg}$ was given. Subsequently, prednisolone (30 mg/day and reducing) and mycophenolate mofetil (500 $\mathrm{mg}$ increased to $1 \mathrm{~g} \mathrm{bd}$ ) were given for acute inflammatory myositis. A trial of cinacalcet was tried as it was felt that primary hyperparathyroidism could be contributing to his hypercalcaemia - his PTH was not completely supressed despite markedly raised calcium levels, that is, $2 \mathrm{mmol} / \mathrm{L}$ (1.6-7.2). The cinacalcet helped lower his calcium levels when he was compliant. He improved symptomatically and metabolically - serum calcium (2.39 mmol/L), eGFR (26) and CK (2505 U/L) in a few months. Despite counselling, he continued to take GH and T on a '6-weeks on/4-weeks off' regime, as he considered these vital for maintaining his body image.

\section{Outcome and follow-up}

His subsequent clinical course was punctuated by repeated admissions for hypercalcaemia and renal deterioration with partial and incomplete recovery on treatment (Table 1). He also presented with an episode of left ventricular failure and was given furosemide $40 \mathrm{mg}$ and bisoprolol $1.25 \mathrm{mg}$. Subsequently, cinacalcet was commenced $-30 \mathrm{mg}$ bd reduced to $30 \mathrm{mg} /$ day. Mycophenolate was stopped due to intolerance and prednisolone was stopped by the patient without consultation. He continued also to be

Table 1 Biochemical indices during acute admissions.

\begin{tabular}{l}
\hline Admission \\
\hline 1 \\
2 \\
3 \\
4 \\
5 \\
\hline
\end{tabular}

\begin{tabular}{c}
\hline eGFR \\
\hline 19 \\
11 \\
16 \\
15 \\
13 \\
\hline
\end{tabular}

\begin{tabular}{c}
\hline Creatinine \\
\hline 298 \\
418 \\
346 \\
362 \\
424 \\
\hline
\end{tabular}

\begin{tabular}{c}
\hline CK \\
\hline 7952 \\
4362 \\
5421 \\
1858 \\
\hline
\end{tabular}

\begin{tabular}{c}
\hline Calcium \\
\hline 3.66 \\
3.34 \\
3.44 \\
3.10 \\
2.88 \\
\hline
\end{tabular}

Biochemical indices during 5 acute admissions demonstrating gradual renal deterioration and improvement in hypercalcaemia and myositis. 
on tamsulosin for prostatic hypertrophy. Despite regular multidisciplinary review, he passed away suddenly. Postmortem examination revealed myocardial infarction as the cause of death.

\section{Discussion}

We have presented a 53-year-old amateur body builder on long-term intermittent $\mathrm{GH}$ and $\mathrm{T}$ self-medication, who presented with severe symptomatic hypercalcaemia complicating acute inflammatory myositis and rhabdomyolysis. He also had AKI (with chronic kidney disease) and significant proteinuria due to FSGS.

We encountered significant diagnostic and management challenges in dealing with him.

(1) the cause for hypercalcaemia -

(i) at admission, a low PTH, severe hypercalcaemia, and phosphate within the reference range suggested possible non-PTH mediated hypercalcaemia (although AKI may have contributed to phosphate retention). The common causes for non-PTH mediated hypercalcaemia were ruled out - for example, normal ACE concentrations; CT/MRI scans did not show chest, abdominal or pelvic masses or lymphadenopathy; muscle biopsy did not show granulomata; Vit D3 level was low (29 nmol/L); absent hyperthyroidism; and normal protein electrophoresis (2). The low active vitamin D3 is likely related to both low 1 alpha hydroxylase activity secondary to his renal dysfunction and low vitamin $\mathrm{D}$ binding protein secondary to nephrotic syndrome. His normal vitamin $\mathrm{D}$ is difficult to explain, but an excessive and persistent exposure to sun may have had a part to play (he routinely wore short sleeved shirts and shorts). Hypervitaminosis A may have been responsible too.

(ii) the likely cause for non-PTH mediated hypercalcaemia in this subject was acute myositis and rhabdomyolysis. A combination of inflammatory myositis (confirmed by (1) high CK, (2) presence of anti Jo-1 and myositis associated antibodies, (3) diagnostic muscle histopathology with mineral deposition, (4) MRI scan appearances and (5) an excellent response to prednisolone), non-traumatic rhabdomyolysis (caused by high intensity exercise as part of his fitness regime), and his use of medications for 'body building' may have all contributed to rhabdomyolysis (4). (iii) detectable (though low) PTH and hypercalcaemia also raises the possibility of a contribution from primary hyperparathyroidism. We were not able to obtain a Sestamibi scan although his neck ultrasound did not show any thyroid or parathyroid related abnormalities.

(iv) raised serum vitamin A levels may also be relevant as a cause for his hypercalcaemia. Vitamin A stimulates osteoclastic resorption and/or inhibit osteoblastic formation (5).

(v) subjects who inject paraffin oil intramuscularly to enhance muscle hypertrophy ('bulking'), as part of a body building regime, develop paraffin oil granulomata which may cause hypercalcaemia mediated by vitamin D3 as reported earlier. However, this subject's vitamin D3 was not diagnostic and there was no muscle granulomata on histopathology.

The prevalence of hypercalcaemia in rhabdomyolysis is quoted to be between 9.2 and $34 \%(6,7,8)$. The variable prevalence is probably related to incomplete identification of these subjects due to the late occurrence of hypercalcaemia (often in the polyuric or recovery phase of AKI) and its mild, often asymptomatic nature. Phosphate retention and PTH resistance cause hypocalcaemia and calcium and phosphate complex deposition in the oliguric early phase of rhabdomyolysis and AKI. However, during the polyuric phase, these calcium and phosphate complexes in muscle are mobilized, causing hypercalcaemia. This subject had widespread radiologically visible (Fig. 1) and histologically proven deposits of calcium in his muscles and soft tissue. He also had continuing muscle damage and rhabdomyolysis causing many episodes of hypercalcaemia - no doubt with contributions from primary hyperparathyroidism, hypervitaminosis A and his continuous GH and T use.

He was given cinacalcet on which his calcium was well controlled during times when he was compliant (9).

(2) the cause for renal dysfunction -

This subject had AKI at admission. But review of previous results indicated gradually declining renal function (eGFR of 79 and $43 \mathrm{~mL} / \mathrm{min}$ several years before) and significant proteinuria for which no cause was obvious then. In the absence of radiological evidence of an obstructive uropathy (despite medication for benign prostatic symptoms), we felt a renal biopsy was indicated because of significant proteinuria and AKI. This showed FSGS without particular diagnostic features of an underlying cause. The tubular 
changes seen were consistent with hypercalcaemia (10). Furthermore, although the initial impression at admission was that increased serum creatinine was due to his large muscle mass (satisfactory eGFR confirmed by isotope GFR), subsequent measurements confirmed deterioration and he was being prepared for possible dialysis. Although toxins such as drugs may cause FSGS, we cannot identify a single cause as being responsible in this gentleman.

(3) the role of hypercalcemia as a cause of constipation -

The mechanism for constipation in hypercalcaemia is not well understood. Calcium activates the endocellular contractile apparatus in striated muscles, but contraction in smooth muscles is dependent on the extra cellular concentrations of calcium as it has less endoplasmic reticulum than striated muscles. It is possible that raised serum calcium causes gut atony due to reduction in neuromuscular excitability (11). The presence of constipation is usually associated with higher serum calcium. The prevalence of constipation in hypercalcaemia was $61.5 \%$ with a serum calcium level of $2.87 \mathrm{mmol}$ as was seen in a study done in Italy in 2012 (11).

\section{Declaration of interest}

The authors declare that there is no conflict of interest that could be perceived as prejudicing the impartiality of the research reported.

\section{Funding}

This research did not receive any specific grant from any funding agency in the public, commercial or not-for-profit sector.

\section{Patient consent}

Written informed consent was obtained from the patient/patient's mother for publication of this case report.

\section{Author contribution statement}

All authors were involved in the diagnosis and management of this subject, either as part of the direct care team or as part of the multidisciplinary team. R R, L D P and M A wrote this manuscript (with contributions from the rest), which was finally approved by all authors.

\section{References}

1 Minisola S, Pepe J, Piemonte S \& Cipriani C. The diagnosis and management of hypercalcaemia.BMJ 2015350 h2723. (https://doi. org/10.1136/bmj.h2723)

2 Coltzman D. Nonparathyroid hypercalemia. Frontiers of Hormone Research 201951 77-90. (https://doi.org/10.1159/000491040)

3 Silva BC, Cusano NE \& Bilezakian JP. Primary hyperparathyroidism. Best Practice and Research: Clinical Endocrinology and Metabolism 2018 32 593-607. (https://doi.org/10.1016/j.beem.2018.09.004)

4 Marcinowska-Suchowierska E, Kupisz-Urbańska M, Łukaszkiewicz J, Płudowski P \& Glenville Jones. Vitamin D toxicity: a clinical perspective. Frontiers in Endocrinology 20189 550. (https://doi. org/10.3389/fendo.2018.00550.2018)

5 Cetari F, Saponaro F, Borsari S \& Marccoci C. Familial and hereditary forms of primary hyperparathyroidism. Frontiers of Hormone Research 201951 40-51. (https://doi.org/10.1159/000491037)

6 Gracia-Maldonado G \& Castro-García RJ. Endocrinological disorders related to the medical use of lithium. A narative review. Revista Colombiana de Psiquiatria 201948 35-43. (https://doi.org/10.1016/j. rcp.2017.01.003)

7 Mirza ZB, Hu S \& Amorosa LF. Bone scintigraphy of severe hypercalcaemia following simvastatin induced rhabdomyolysis. Clinical Cases in Mineral and Bone Metabolism 201613 257-261. (https://doi.org/10.11138/ccmbm/2016.13.3.257)

8 Wang FY, NG CY, Wu J, Kuo KL, Chang YY \& Kuo TT. Acquired perforating calcific collagenosis in a drug addict with rhabdomyolysis and transient hypercalcaemia. Journal of Cutaneous Pathology 201946 84-87. (https://doi.org/10.1111/cup.13371)

9 Boyce BF, Fell GS, Elder HY, Junor BJ, Elliot HL, Beastall G, Fogelman I \& Boyle IT. Hypercalcaemic osteomalacia due to aluminium toxicity. Lancet 19822 1009-1013. (https://doi. org/10.1016/s0140-6736(82)90049-6)

10 Jose N \& Kurian GP. Schimidt syndrome: an unusual cause of severe hypercalcemia. Journal of Clinical and Diagnostic Research 201610 OD21-2. (https://doi.org/10.7860/JCDR/2016/16770.7783)

11 Ragno A, Pepe J, Badiali D, Minisola S, Romagnoli E, Severi C \& D'Erasmo E. Chronic constipation in hypercalcaemic patients with primary hyperparathyroidism. European Review for Medical and Pharmacological Sciences 201216 884-889.

Received in final form 31 May 2020

Accepted 15 June 2020 